\title{
Sequías en Chile central a partir de diferentes índices en el período 1981-2010
}

\author{
Pablo Sarricolea ${ }^{1,2}$, psarricolea@uchilefau.cl; Óliver Meseguer-Ruiz ${ }^{2}$
}

\begin{abstract}
RESUMEN
Se analizan las diferentes dimensiones de la sequía meteorológica en Chile central con el propósito de caracterizar su ocurrencia a múltiples escalas temporales y encontrar indicios de cambio en su variabilidad y frecuencia. Para ello se ha dispuesto de 27 estaciones meteorológicas a resolución diaria, las cuales permiten identificar períodos secos bajo el umbral 0,1 mm. Además de establecer las rachas de sequías y su persistencia (mediante el índice de Besson), se aplicó el índice estándar de precipitación (SPI) para conocer la varibilidad internanual de la sequía, justamente en una región de clima semiárido y mediterráneo. Los resultados indican mayor persistencia de días secos en la región de Valparaíso, lo cual es consistente con la longitud de las rachas de sequía, en muchos casos superior a 22 días al año. El evento La Niña en el invierno de 1998 provocó la mayor racha de días secos del trimestre lluvioso (67 días), sumando en total 86 días sin lluvia. No obstante, no se ha podido probar cambios en su variabilidad y frecuencia.
\end{abstract}

Palabras clave: Chile central, clima mediterráneo, índice de Besson, precipitación diaria, sequía.

\section{Droughts in central Chile using different indices during the 1981-2010 period}

\begin{abstract}
The different dimensions of meteorological drought in central Chile in order to characterize their occurrence at multiple time scales and find evidence of variability and change in frequency are analyzed. For that, there are 27 meteorological stations at daily resolution arranged, which allow the identification of dry periods under the threshold of $0.1 \mathrm{~mm}$. Besides establishing dry spells and its persistence (using the Besson index), the standard precipitation index (SPI) was applied to determine the internanual varibilidad of drought, precisely in a region with semiarid and Mediterranean climate. The results show greater persistence of dry days in the region of Valparaíso, which is consistent with the length of dry spells in many cases exceeding 22 days a year. The event La Niña in the winter of 1998 caused the longest streak of dry days of the rainy trimester (67 days), totaling 86 days without rain. However, it has not been able to prove their variability and changes in frequency.
\end{abstract}

Keywords: Besson index, central Chile, diary precipitation, drought, Mediterranean climate.

Recibido el 4 de diciembre de 2014, aceptado el 6 de mayo de 2015.

Departamento de Geografía, Universidad de Chile, Portugal 84, Santiago, Chile.

2 Grupo de Climatología, Departamento de Geografía Física y A.G.R., Universidad de Barcelona, España. 


\section{INTRODUCCIÓN}

La sequía es un extremo hidrometeorológico de efectos acumulativos, afectando a la sociedad y los sistemas físicos naturales. Posee una extensión temporal muy diferente a otros extremos hidrometeorológicos, pues mientras las precipitaciones abundantes se registran en pocas horas o días, las sequías pueden extenderse durante meses o años.

La sequía representa un problema multidimensional, ya que afecta a la economía, los sistemas ecológicos, la sociedad y la generación de energía hidroeléctrica. Debido a ello, la sequía presenta diversas definiciones que van más allá de la propia condición de ausencia de lluvia o sequía meteorológica. Más aún, las sociedades han encontrado mecanismos para declarar la situación de sequía y con ello, mecanismos de gestión, muchas veces plasmados en los códigos de agua.

Según BELLOW et al. (2007), entre 1900 y 2004 la sequía fue el riesgo natural que provocó las mayores pérdidas humanas (54\%), afectando a un tercio de la población mundial (sólo detrás de inundaciones que agrupa al $51 \%$ ) y ocupando el cuarto lugar en las pérdidas económicas, las cuales son lideradas por los huracanes, inundaciones y terremotos.

El interés de estudiar la sequía en Chile central (región de Valparaíso y Metropolitana) se debe a que en ella se emplaza gran parte de la agricultura y los centros poblados, por lo que la disponibilidad de agua es un factor estratégico para el desarrollo. El área de estudio presenta una alta vocación de producción agrícola y vitivinícola, lo que redunda en una fragilidad ante el desastre de la sequía.

En las últimas décadas han ocurrido varios episodios de sequía en Chile central. Varias comunas han sido declaradas como zonas de catástrofe por sequía y otras están muy cerca de entrar en dicha categoría. Según datos del BANCO MUNDIAL (2011), entre las regiones de Arica Parinacota hasta la Metropolitana, la demanda de agua supera a la oferta hídrica, por lo que el área de estudio es de gran interés. Más aún, en Chile central, en el contexto de cambio climático, se pronostican alzas de las temperaturas $\left(2^{\circ}\right.$ a $\left.4^{\circ} \mathrm{C}\right)$ y dismunuciones cercanas al $30 \%$ en los montos de precipitación (FUENZALIDA et al. 2007, BRAVO et al. 2014).

Los períodos secos y lluviosos en Chile central están asociados a los centros de acción dominantes en superficie, en este caso el Anticiclón del Pacífico Sur Oriental (APSO) y el Cinturón de Bajas Presiones Subpolares (CBPP), más las configuraciones en altura (vaguadas, dorsales, altas y bajas segregadas). A ellas se debe sumar el efecto sobre las precipitaciones que confieren los patrones de variabilidad de baja frecuencia. Para Chile central se distinguen El Niño Oscilación del Sur (ENSO), la Oscilación Decadal del Pacífico (PDO) y la Oscilación Antártica (AAO) (SARRICOLEA et al., 2014).

QUINTANA \& ACEITUNO (2006) señalan que existe una clara variabilidad interdecadal en el régimen de precipitación en Chile central, lo cual parece estar relacionado con los cambios en la intensidad del APSO, afectado por la PDO y el ENSO (SARRICOLEA et al., 2014). Así, las tendencias a la disminución de las precipitaciones (sequía) coinciden con una significativa intensificación del APSO, un Índice de Oscilación del Sur (SOI) positivo y una fase fría de la PDO. Por el contrario, un debilitamiento del anticiclón conlleva un aumento de las precipitaciones, fase negativa del SOI (Episodios El Niño) y una fase cálida de la PDO (QUINTANA \& ACEITUNO, 2012). Por último, la relación entre las sequías en Chile central y las distintas fases de la Oscilación Antártica (AAO) (THOMPSON \& WALLACE, 
2000) permiten asociar que durante las fases positivas del índice se presenta una menor posibilidad de actividad frontal en Chile central, y, por lo tanto, menos precipitaciones.

Según BRAVO et al. (2014), los modelos del INTERGOVERNMENTAL PANEL ON CLIMATE CHANGE (IPCC 2013) aplicados a Chile central (en su escenario más pesimista RCP8.5) señalan que se podrían triplicar las sequías y con ello las de carácter bianual y de 3 años o más.Con ello, la sequía adquire una importancia fundamental en las actividades económicas que se desarrollan en esta región, al igual que el abastecimiento de agua para consumo.

Así, en este artículo se abordan tres metodologías de cálculo matemático y estadístico que apuntan a establecer la distribución espacial y la magnitud de los períodos de sequía meteorológica, con el fin de transformarse en una herramienta que permita reducir la incertidumbre frente a los desastres de sequía. Junto a lo antes mencionado se realiza un análisis de las teleconexiones climáticas para entender su relación con los períodos de escasez de precipitaciones en el área de estudio.

\section{MATERIALES Y MÉTODOS}

El área de estudio abarca administrativamente a la V Región y la XIII Región, correspondientes a Valparaíso y Metropolitana. Estas regiones poseen una ubicación geográfica aproximada entre los $32^{\circ} 01^{\prime}$ y los $34^{\circ} 08^{\prime}$ de latitud Sur y entre los $71^{\circ} 45^{\prime}$ y $69^{\circ} 46^{\prime}$ de longitud Oeste, y una superficie de $31.387,02 \mathrm{Km}^{2}$. Posee un clima transicional entre el semiárido y el mediterráneo, una alta concentración de población y actividades productivas, las cuales demandan intensivamente agua, y son afectadas por anomalías climáticas de distinta naturaleza, en especial por teleconexiones tales como ENSO (evaluada mediante el Índice Oceánico de El Niño),
PDO y AAO, las cuales modulan períodos secos y lluviosos.

En total se han utilizado 27 estaciones meteorológicas (ver Tabla 1), las que se sitúan entre los 25 y 2.575 metros sobre el nivel del mar. Todas ellas cubren el período 1981-2010, a excepción de la Estación Valparaíso que abarca entre 1981-2005. Su localización espacial se puede ver en la Figura 1.

Se ha aplicado el índice de Besson y rachas de sequía a resolución diaria; y a resolución semestral, el índice estandarizado de precipitación (SPI).

a) Índice de Besson (SB): es una medida indicativa de la persistencia de la sequía (o lluvia). Fue ideado por BESSON (1924), y su fórmula de cálculo es la siguiente:

$$
\mathrm{SB}=\frac{P_{0}(0)-P(0)}{1-P(0)}
$$

SB es un cociente, donde $P_{0}(0)$ es la probabilidad de día seco después de un día seco, y es la probabilidad de día seco en función del total de días.

Como es sabido, la persistencia de SB depende que $P_{0}(0) \geq P(0)$ y siempre se verifica que $0 \leq P(0) \leq 1$. SB es el coeficiente de dos números reales positivos, siendo siempre el numerador menor o igual que el denominador, por lo cual los valores de SB pertenecen al intervalo entre 0 y 1 . Los valores cercanos a 1 en el índice de Besson indican mucha persistencia de sequía y los valores próximos a 0 significan poca persistencia de días secos. 
Tabla 1. Estaciones Meteorológicas CONSIDERADAS SEgúN LOCALIZACiÓN, LONGITUd DE LA SERIE DIARIA, AÑOS Y FUENTE.

Table 1. Meteorological stations considered by location, Length of THE DaILy SERies, Years AND SOURCE.

\begin{tabular}{|c|c|c|c|c|c|c|c|}
\hline $\mathrm{N}^{\circ}$ & Estación & Latitud (S) & Longitud (W) & Altitud (m) & Serie & Años & Fuente \\
\hline 1 & Pedernal Hacienda & $-32,09$ & $-70,80$ & 1100 & $1981-2010$ & 30 & DGA \\
\hline 2 & Quilimarí & $-32,12$ & $-71,50$ & 25 & $1981-2010$ & 30 & DGA \\
\hline 3 & Chalaco Hacienda & $-32,19$ & $-70,79$ & 880 & $1981-2010$ & 30 & DGA \\
\hline 4 & El Trapiche & $-32,23$ & $-70,71$ & 1180 & $1981-2010$ & 30 & DGA \\
\hline 5 & Palquico & $-32,25$ & $-71,14$ & 450 & $1981-2010$ & 30 & DGA \\
\hline 6 & Alicahue Hacienda & $-32,34$ & $-70,75$ & 750 & $1981-2010$ & 30 & DGA \\
\hline 7 & Estero Rabuco & $-32,85$ & $-71,12$ & 300 & $1981-2010$ & 30 & DGA \\
\hline 8 & Vilcuya & $-32,86$ & $-70,47$ & 1100 & $1981-2010$ & 30 & DGA \\
\hline 9 & Riecillos & $-32,93$ & $-70,35$ & 1290 & $1981-2010$ & 30 & DGA \\
\hline 10 & Caleu & $-33,01$ & $-70,99$ & 1150 & $1981-2010$ & 30 & DGA \\
\hline 11 & Rungue Embalse & $-33,02$ & $-70,91$ & 700 & $1981-2010$ & 30 & DGA \\
\hline 12 & Valparaíso & $-33,06$ & $-71,61$ & 41 & $1981-2005$ & 25 & $\mathrm{DMC}$ \\
\hline 13 & Lago Peñuelas & $-33,15$ & $-71,54$ & 360 & $1981-2010$ & 30 & DGA \\
\hline 14 & Colliguay & $-33,17$ & $-71,15$ & 490 & $1981-2010$ & 30 & DGA \\
\hline 15 & Cerro Calán & $-33,40$ & $-70,54$ & 800 & $1981-2010$ & 30 & DGA \\
\hline 16 & Quinta Normal (Santiago) & $-33,41$ & $-70,79$ & 527 & $1981-2010$ & 30 & $\mathrm{DMC}$ \\
\hline 17 & Los Panguiles & $-33,44$ & $-71,03$ & 195 & 1981-2010 & 30 & DGA \\
\hline 18 & $\begin{array}{c}\text { Terraza Oficina Central } \\
\text { DGA }\end{array}$ & $-33,45$ & $-70,65$ & 560 & $1981-2010$ & 30 & DGA \\
\hline 19 & Antupirén & $-33,50$ & $-70,52$ & 920 & $1981-2010$ & 30 & DGA \\
\hline 20 & San Antonio & $-33,57$ & $-71,62$ & 150 & $1981-2010$ & 30 & DGA \\
\hline 21 & Cerrilos de Leyda & $-33,63$ & $-71,51$ & 180 & $1981-2010$ & 30 & DGA \\
\hline 22 & San José de Maipo & $-33,64$ & $-70,35$ & 970 & $1981-2010$ & 30 & DGA \\
\hline 23 & Pirque & $-33,67$ & $-70,59$ & 670 & $1981-2010$ & 30 & DGA \\
\hline 24 & El Yeso Embalse & $-33,68$ & $-70,09$ & 2575 & $1981-2010$ & 30 & DGA \\
\hline 25 & Melipilla & $-33,68$ & $-71,20$ & 170 & $1981-2010$ & 30 & DGA \\
\hline 26 & Carmen de las Rosas & $-33,76$ & $-71,15$ & 165 & $1981-2010$ & 30 & DGA \\
\hline 27 & San Gabriel & $-33,78$ & $-70,24$ & 1240 & $1981-2010$ & 30 & DGA \\
\hline
\end{tabular}




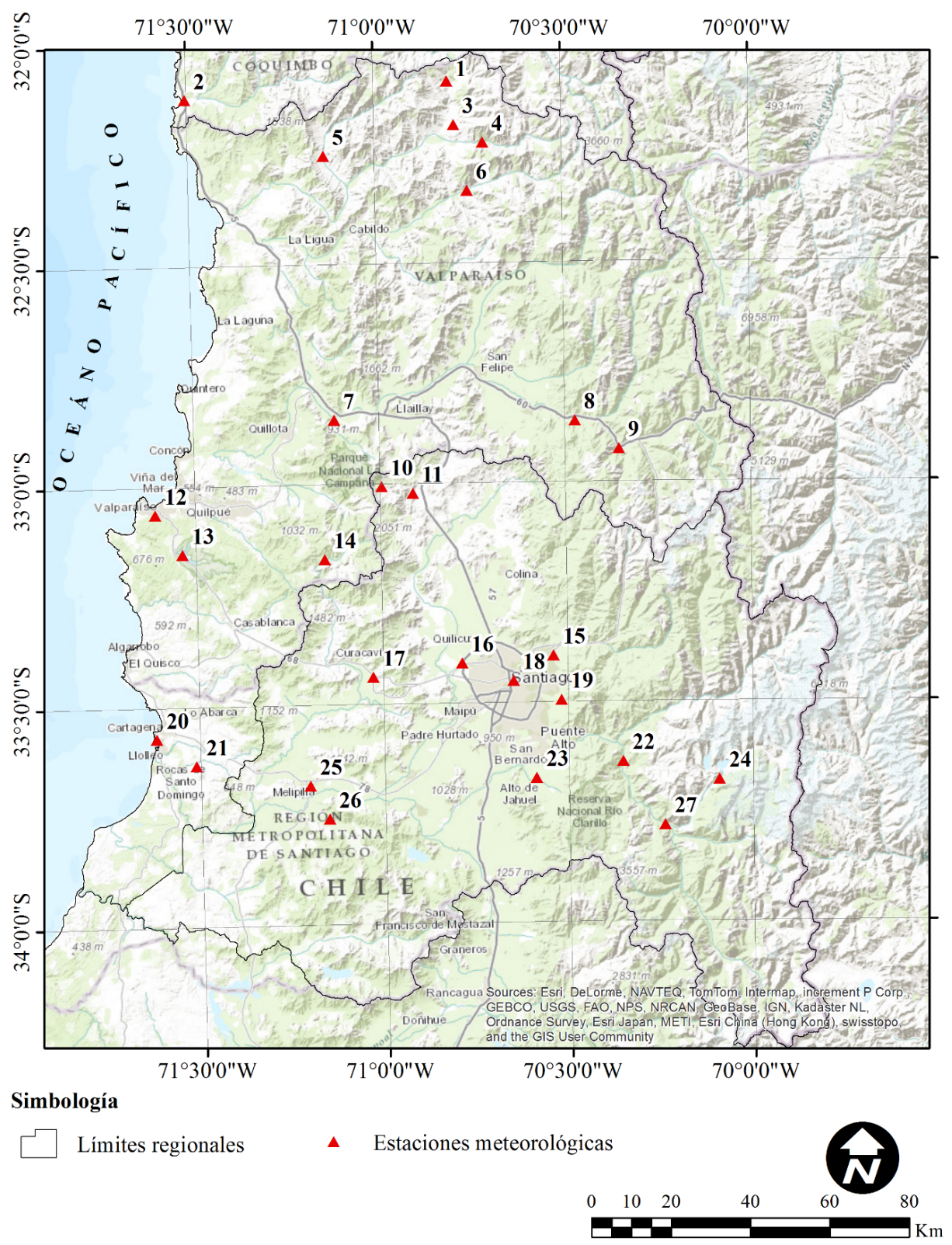

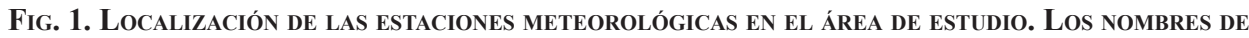
las estaciones aparecen en la Tabla 1 SEgún El NúMero.

Fig. 1. Location of meteorological Stations in STUdy area. The names of the Meteorological STATIONS ARE SHOWN IN TABLE 1 ACCORDING TO THE NUMBER. 
También se puede decir que, independientemente de que llueva mucho o poco en un determinado lugar, un valor alto de SB significa poca probabilidad que la sequía se produzca en días aislados, y un valor pequeño de SB confirma que existe una importante tendencia a que la sequía se produzca en días aislados (PÉREZ et al. 1986).

b) Método de las rachas: se trata de un período constituido por días que presentan un mismo carácter en el estado meteorológico (MARTÍNVIDE 2003); En el caso de estudio, jornadas consecutivas sin registro de precipitación. La aplicación de este método consiste en contabilizar el número absoluto de rachas o secuencias de días secos durante el período de estudio, así como cuantificar la longitud promedio, máxima y mínima absoluta de las mismas. Para ello, previamente se deben haber agrupado las jornadas de días secos en rachas. El cálculo de la longitud media de las rachas de sequía $\left(l_{1}\right)$ se obtiene como cociente entre el total de días $\operatorname{secos}(\mathrm{L})$ y el total de rachas (NR) del período de estudio considerado.

$$
l_{1}=L / N R
$$

c) El Índice Estándar de Precipitación (SPI): está diseñado para mejorar la detección del comienzo de la sequía y para realizar su seguimiento (MCKEE et al. 1993). El SPI es una medición de la sequía muy simple, pues se basa solamente en las probabilidades de ocurrencia de precipitación/sequía en un tiempo específico. Una característica importante del SPI es la flexibilidad para medir la sequía mutiescalarmente, desde el punto de vista temporal. Debido a que las sequías tienen una gran variación en su duración, es importante detectarlas y monitoriarlas en una amplia variedad de escalas temporales. Las sequías de corto término son medidas por instrumentos meteorológicos y son definidas de acuerdo a la climatología local específica. A su vez, las sequías de importancia para la agricultura señalan déficit de la humedad del suelo, siendo sus mejores representantes aquellas de tres a seis meses. Las sequías más prolongadas (de semestres a años) pueden alcanzar impactos significativos sobre las reservas de agua, tanto superficial y como subterránea.

Los valores de SPI se obtienen mediante la relación estandarizada de las precipitaciones durante un intervalo de tiempo específico (promedios de 1, 3 o 6 meses), es decir, se usan puntajes Z. Los valores varían desde 3 a -3 . La Tabla 2 muestra los valores de clasificación del SPI.

Tabla 2. Valores y Categorías de SEQUía ASIGNAdOS AL Í ÍNDICE EsTÁNDAR DE Precipitación (SPI).

TABLE 2. VAlues AND CATEGORIES OF DROUGHT assigned to the Standard Precipitation IndeX (SPI).

\begin{tabular}{|c|c|}
\hline Valores del SPI & Categoría de la sequía \\
\hline 2,00 o más & Extremadamente húmedo \\
\hline 1,50 a 1,99 & Muy húmedo \\
\hline 1,00 a 1,49 & Moderadamente húmedo \\
\hline$-0,99$ a 0,99 & Casi normal \\
\hline$-1,00$ a $-1,49$ & Moderadamente seco \\
\hline$-1,50$ a $-1,99$ & Severamente seco \\
\hline$-2,00$ o menos & Extremadamente seco \\
\hline
\end{tabular}

Para alcanzar una mejor representación espacial de los índices de sequía se utilizan técnicas de regresión lineal múltiple, basadas en variables tales como la latitud, longitud, altitud, precipitación anual. En los casos que la estadística lineal no entregó resultados significativos se usó interpolación no lineal (Spline de tensión con factor 0,4), el cual mejora la representación espacial y es provista del mismo sistema de información geográfica, en este caso de ArcGis 10.2. 


\section{RESULTADOS}

El índice de Besson posee un promedio para el área de estudio de 0,46, siendo Los Panguiles la estación con el valor más bajo $(0,337)$ y Estero Rabuco la estación que marca el máximo SB $(0,69)$, tal como se aprecia en la Tabla 3.

La configuración espacial del índice de Besson (Fig. 2) indica una mayor persistencia de días secos en la Región de Valparaíso (litoral norte y Cordillera de los Andes) y sotavento de la Cordillera de la Costa al sur de la Región Metropolitana (Alhue). Aquellas áreas menos expuestas a sequía diaria según Besson, son la ciudad de Valparaíso, la depresión intermedia y parte de ambas cordilleras en la Región Metropolitana.

Tabla 3. Índice de Besson en base a la Probabilidad de Persistencia de día Seco luego de UN día SECo $\left(P_{0}(0)\right)$ Y LA PERSISTENCIA de día SECo $(P(0))$ PARA LAS ESTACIONES METEOROLÓGICAS ESTUDIADAS.

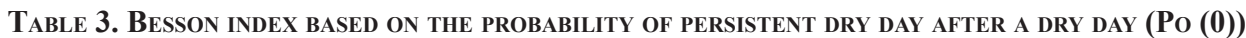
AND PERSISTENT DRY DAY (P (0)) FOR METEOROLOGICAL STATIONS STUDIED.

\begin{tabular}{|c|c|c|c|c|}
\hline $\mathrm{N}^{\circ}$ & Estación & $P_{0}(0)$ & $P(0)$ & Índice Besson (SB) \\
\hline 1 & Pedernal Hacienda & 0,958 & 0,916 & 0,498 \\
\hline 2 & Quilimarí & 0,969 & 0,920 & 0,616 \\
\hline 3 & Chalaco Hacienda & 0,961 & 0,918 & 0,520 \\
\hline 4 & El Trapiche & 0,956 & 0,921 & 0,450 \\
\hline 5 & Palquico & 0,960 & 0,922 & 0,490 \\
\hline 6 & Alicahue Hacienda & 0,957 & 0,932 & 0,369 \\
\hline 7 & Estero Rabuco & 0,958 & 0,862 & 0,693 \\
\hline 8 & Vilcuya & 0,943 & 0,899 & 0,437 \\
\hline 9 & Riecillos & 0,944 & 0,866 & 0,583 \\
\hline 10 & Caleu & 0,956 & 0,920 & 0,455 \\
\hline 11 & Rungue Embalse & 0,953 & 0,897 & 0,544 \\
\hline 12 & Valparaíso & 0,952 & 0,923 & 0,374 \\
\hline 13 & Lago Peñuelas & 0,942 & 0,900 & 0,423 \\
\hline 14 & Colliguay & 0,951 & 0,900 & 0,504 \\
\hline 15 & Cerro Calán & 0,930 & 0,869 & 0,467 \\
\hline 16 & Quinta Normal (Santiago) & 0,929 & 0,890 & 0,357 \\
\hline 17 & Los Panguiles & 0,940 & 0,909 & 0,337 \\
\hline 18 & Terraza Oficina Central DGA & 0,934 & 0,882 & 0,441 \\
\hline 19 & Antupirén & 0,943 & 0,905 & 0,406 \\
\hline 20 & San Antonio & 0,936 & 0,884 & 0,448 \\
\hline 21 & Cerrilos de Leyda & 0,941 & 0,905 & 0,380 \\
\hline 22 & San José de Maipo & 0,941 & 0,897 & 0,421 \\
\hline 23 & Pirque & 0,936 & 0,889 & 0,419 \\
\hline 24 & El Yeso Embalse & 0,929 & 0,881 & 0,402 \\
\hline 25 & Melipilla & 0,941 & 0,901 & 0,405 \\
\hline 26 & Carmen de las Rosas & 0,955 & 0,901 & 0,540 \\
\hline 27 & San Gabriel & 0,945 & 0,896 & 0,468 \\
\hline
\end{tabular}


Respecto a los extremos de rachas medias de sequía, se tiene que ellas fluctúan entre 14 y 32 días (Embalse El Yeso y Quilimarí), siendo muy dependientes del incremento latitudinal y altitudinal. La confección de la cartografía fue mediante una regresión lineal múltiple, la cual alcanzó un coeficiente de correlación de $0,84 \quad\left(R^{2}=0,70\right)$, siendo los valores estadísticamente significativos (p-valor igual a 0,000$)$, usando tres variables: altitud (metros), latitud (grados decimal negativos) y precipitación anual (milímetros). La altitud corresponde al valor de cada píxel de la misión topográfica radar Shutlle (Shuttle Radar Topography Mission) o SRTM de resolución de 90 metros, y la precipitación anual a la modelada por PLISCOFF et al. (2014).

Rachas de sequía $=250,063+$ Altitud $\times$ $-0,002+$ Latitud $\times 6,990+$ Pp Anual $\times 0,006$

Los resultados de la ecuación implican que por ejemplo, el incremento de la latitiud en un grado disminuye la longitud de la sequía en 7 días, o que aumentar la altitud en 500 metros las disminuye en un día, siendo la precipitación anual la variable menos sensible, pues 100 milímetros más de lluvia suponen medio día más de sequía. En este sentido, la cantidad de precipitación media parece la variable más compleja de analizar en función de las rachas de sequía, pero como su importancia en la ecuación es menor e intervienen otras variables (latitud y altitud) se compensan mutuamente.

Al examinar las longitudes máximas de las rachas de sequía para una estación (Quinta Normal), es posible identificar dos antecedentes muy relevantes. En primer lugar, las rachas mayores a 120 días (de verano y otoño) se han presentado en 14 de los 30 años de la serie analizada: 1984, 1986, 1988, 1989, 1990, 1993, 1994, 1999, 2001, 2002, 2004, 2006, 2009 y 2010, lo cual permite afirmar que la última década ha sido más seca que las dos precedentes, aumentando las sequías de 120 días de $40 \%$ a $60 \%$. En segundo lugar, las sequías mayores de 30 días en invierno han ocurrido en cinco años bien concretos: 1986, 1989, 1993, 1995 y 1998; este último año con dos períodos que resultan del invierno con menos días de lluvia (4 en total y 86 días secos), sumando un total de 23,4 mm. De esta manera, las rachas medias muestran las peores cifras en la región de Valparaíso (Fig. 3), pues en promedio superan los 20 días sin lluvia. El gradiente de las rachas de sequía posee un componente latitudinal muy notorio al ver la Figura 3, indicando una disminución de la longitud de días secos con el aumento de la latitud.

Respecto al SPI de 6 meses se han encontrado muchas diferencias intraanuales del comportamiento espacial de la sequía (Tabla 4). Por ejemplo hay años muy contrastados, lo que significa que algunos observatorios marcan alguna categoría de sequía o humedad mientras que el resto de estaciones está en el margen de la "normalidad". Esto confirma que espacialmente esta región posee un importante control topoclimático.

Para entender de mejor manera el comportamniento de las sequías (y las precipitaciones) se ha correlacionado el SPI durante 6 meses con los índices de teleconexión ONI, AAO y PDO, con y sin desfase. Se ha detectado que sin desfase las correlaciones son mejores para el índice ONI, y con un mes de desfase (hacia atrás) la AAO y PDO mejoran levemente sus resultados. No obstante, los resultados son significativos por sobre el 95\% sólo para el Índice Oceánico de El Niño, mostrando una relación directa entre la presencia semestres El Niño con lluvias abundantes y sequías con La Niña, tal como revela la Figura 4. 


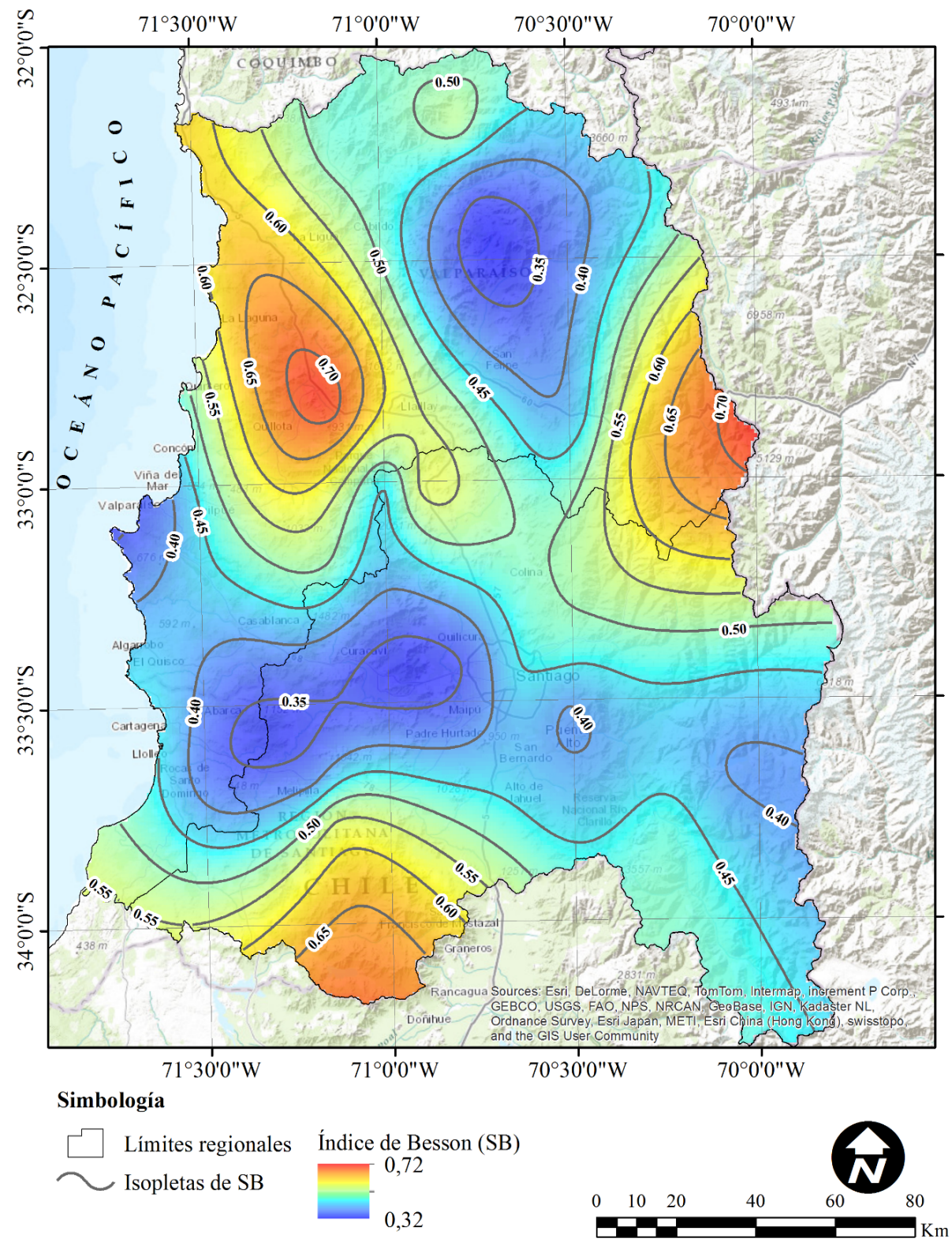

Fig. 2. Distribución espacial del índice de Besson en las Regiones de Valparaíso y Metropolitana, Período 1981-2010.

Fig. 2. Spatial distribution of index Besson in Regions of Valparaiso and Metropolitan, PERIOD 1981-2010. 


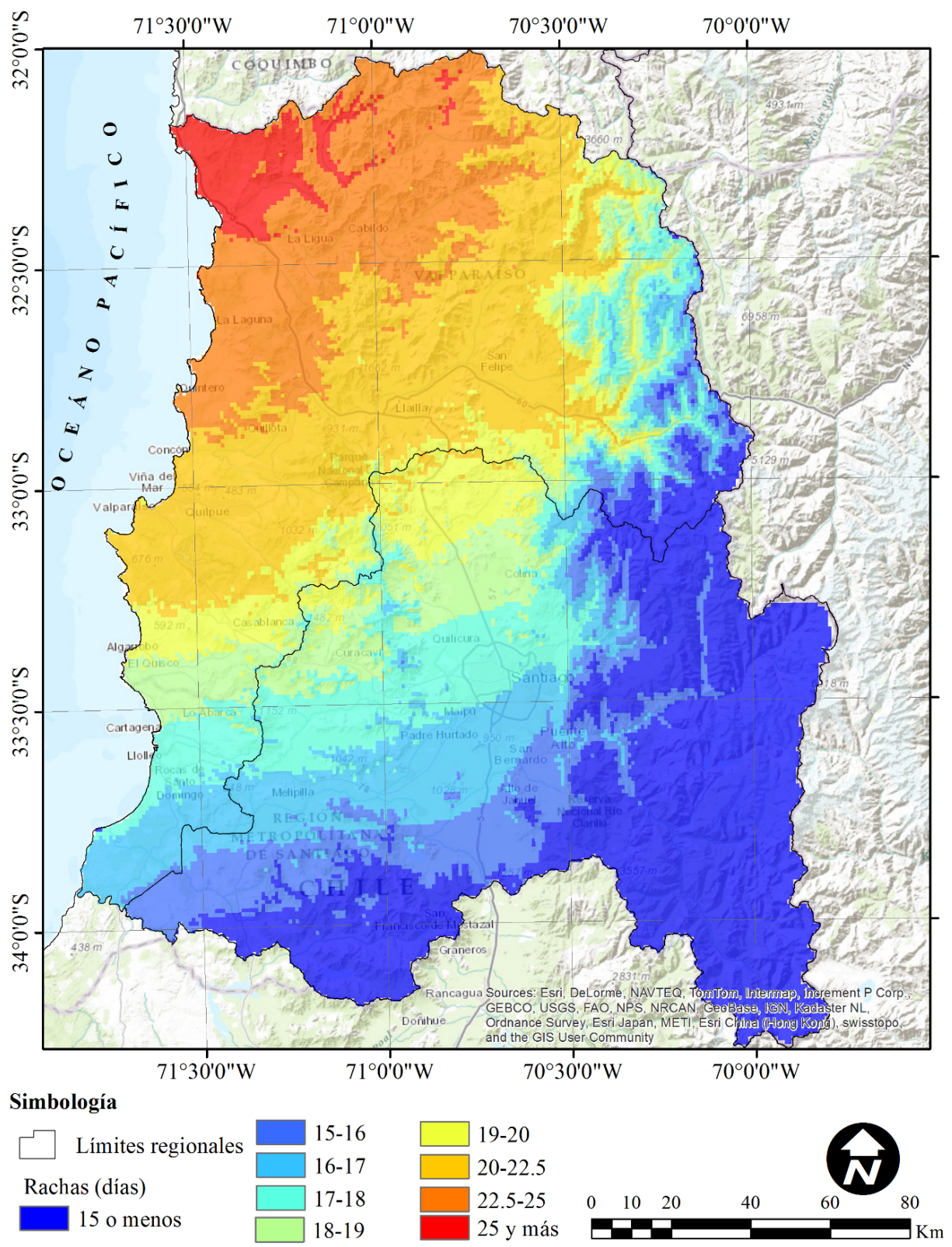

Fig. 3. Distribución espacial de la longitud de las Rachas de Sequía en las Regiones de Valparaíso y Metropolitana, Período 1981-2010.

Fig. 3. Spatial distribution of the length of dry spells of Valparaiso and Metropolitan, PERIOD 1981-2010. 
Tabla 4. Categorías en COlor del índice estandarizado de PRecipitaciones (SPI) de SeIS Meses PARA LAS 27 ESTACIONES ESTUDIADAS, PERÍODO 1981-2010.

Table 4. Color Categories of STANDARDized PRECiPITATION (SPI) SIX MONTHS FOR the 27 STATIONS STUDIED, PERIOD FROM 1981 TO 201.

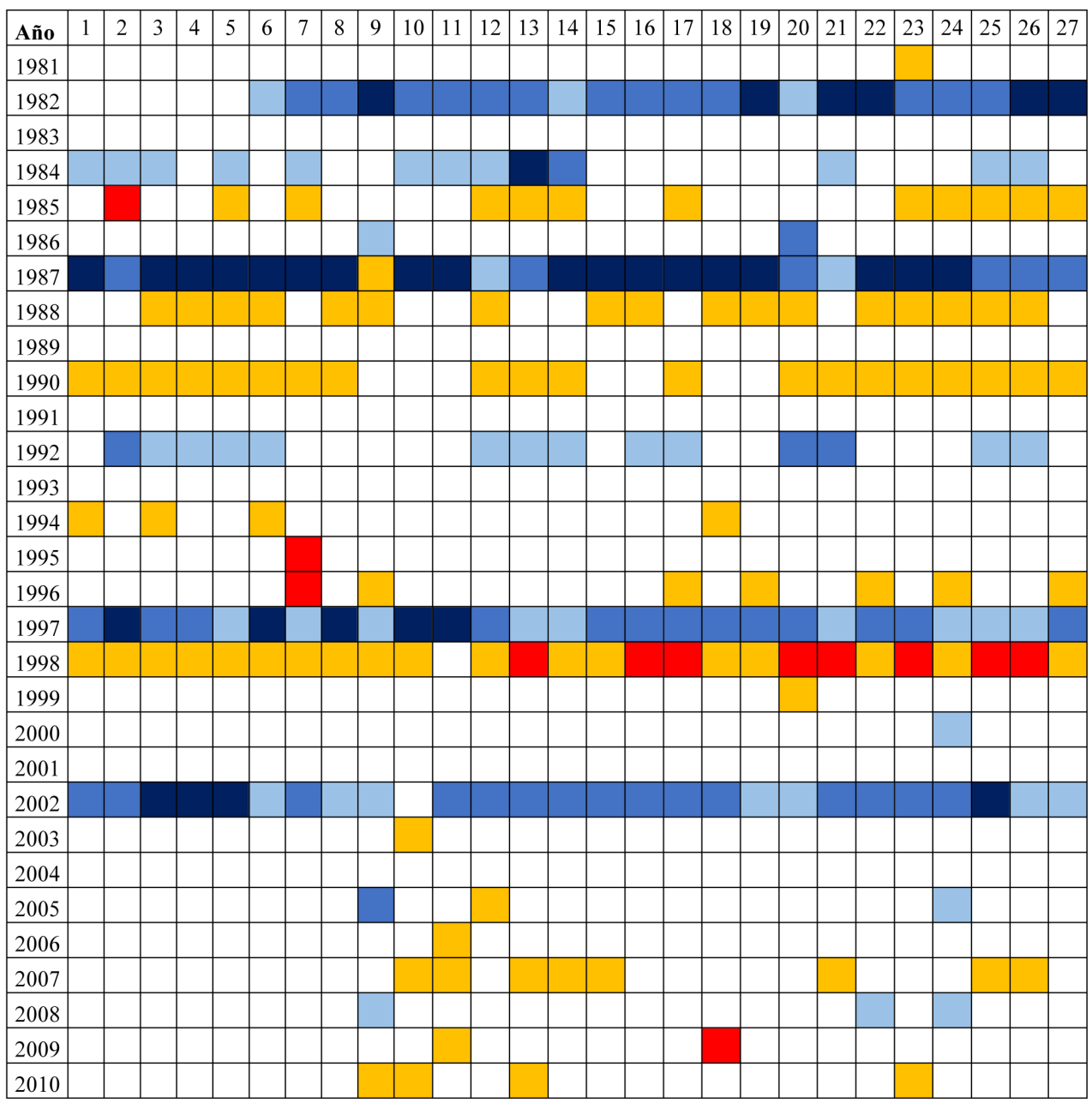

El color rojo indica sequía severa, naranjo sequía leve, celeste moderadamente húmedo, azul muy húmedo y azul oscuro extremadamente húmedo. 


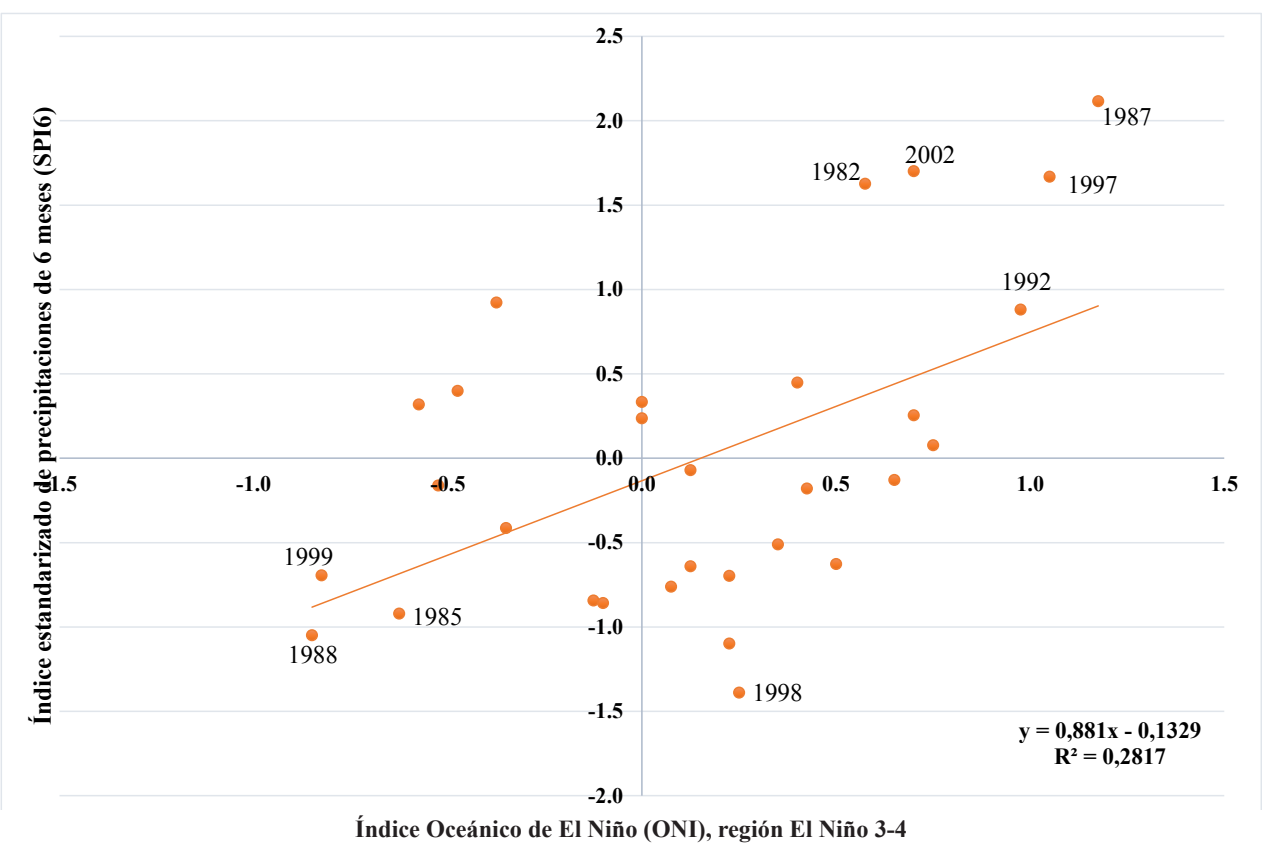

Fig. 4. Correlación entre el SPI6 y el ONI del mismo período sin desfase.

Fig. 4. Correlation between SPI6 and ONI the same Period without lag.

\section{CONCLUSIONES}

Las distribuciones espaciales de los distintos índices de sequía son de gran ayuda para definir los territorios (subcuencas, áreas agroclimáticas y terrenos de cultivo) que son más afectados por episodios adversos y podrían servir de apoyo a las organizaciones de usuarios de agua para enfrentar de mejor manera sus efectos.

Los resultados del índice de Besson permiten concluir que las cuencas más afectadas por la sequía se encuentran mayoritariamente en la región de Valparaíso, específicamente en las comunas administrativas de $\mathrm{La}$ Ligua, Petorca y Cabildo, y también en la región Metropolitana, mayoritariamente en la cuenca del Estero Alhué (Provincia de Melipilla). Esto no sigue necesariamente patrones geográficos latitudinales generales, pues las primeras cuencas desembocan al Océano Pacífico y Alhué es una cuenca a sotavento, lo cual confiere características muy particulares.

Según el análisis del índice SPI de 6 meses, se puede determinar que entre marzo y agosto (MAMJJA) la sequía es leve en un $40 \%$ del tiempo; y moderada a severa en un $7 \%$ y $3 \%$, respectivamente. Esto se explica porque los meses de más precipitación son los que poseen mayor variabilidad interanual.

Las zonas donde la sequía impacta de menor manera (o son más resistentes) corresponden a precordillera y cordillera ubicadas entre las regiones de Valparaíso y Metropolitana. Esto se aprecia en la distribución espacial del SPI mínimos en años La Niña pues son menos afectados por la sequía. Además, en la distribución espacial del SPI máximo en años El Niño, precordillera y cordillera son más lluviosos y deben ser considerados como reservorios de agua. También, esta situación se evidencia en las rachas de 
sequía, mostrando menores longitudes medias de rachas de sequía en el sector antes mencionado.

Existe una relación entre la manifestación de La Niña y la sequía, la cual se ha comprobado estadísticamente.

Lo relevante es que después de períodos largos de sequía, como el que manifiesta actualmente el país, toman mayor importancia los aportes de los glaciares $\mathrm{y}$ acuíferos para disponer de agua en los períodos de primavera y verano. Es decir, si Chile pierde superficie glaciar o recarga de nuestras napas freáticas, la vulnerabilidad y disponibilidad de agua va a ser absolutamente insostenible.

\section{AGRADECIMIENTOS}

A la Dirección General de Aguas (DGA) por facilitar la información pluviométrica utilizada. A FONDECYT Nº 11130629 y la Vicerrectoría de Investigación y Desarrollo (VID) de la Universidad de Chile (U-APOYA, código CSSOC-2012-292), ambas por financiar esta investigación. Al Grupo de Climatología de la Universidad de Barcelona (2014-SGR300).

\section{REFERENCIAS}

BANCO MUNDIAL, 2011. Diagnóstico de la gestión de los recursos hídricos en Chile. Departamento de Medio Ambiente y Desarrollo Sostenible de la Región para América Latina y el Caribe. 81 pp.

BELOW R., E. GROVER-KOPEC \& M. DILLEY, 2007. Documenting droughtrelated disasters a global reassessment. The Journal of Environment \& Development, 16(3): 328-344.

BESSON, L., 1924. On the probability of rain. Monthly Weather Review. 52, 308.
BRAVO, M., R. FLORES, R. GALINDO, R. GARREAUD, E. MUÑOZ, A. SEREY \& M. VIALE, 2014. Determinación de posibles impactos en la gestión de los abastecimientos humanos de agua situados en la zona Metropolitana de Chile, provocados por fenómenos asociados a cambio climático. Aquaea Paper $\mathrm{N}^{\circ} 5$. Fundación Aquaea. 68 pp.

FUENZALIDA, H., P. ACEITUNO, M. FALVEY, R. GARREUAD, M. ROJAS, \& R. SÁNCHEZ, 2007. Study on climate variability for Chile during the 21 st century. Technical Report prepared for the National Environmental Committee, Spanish, Santiago.

INTERGOVERNMENTAL PANEL ON CLIMATE CHANGE (IPCC), 2013. Climate Change 2013: The Physical Science Basis. Contribution of Working Group I to the Fifth Assessment Report of the Intergovernmental Panel on Climate Change [Stocker, T.F., D. Qin, G.K. Plattner, M. Tignor, S.K. Allen, J. Boschung, A. Nauels, Y. Xia, V. Bex and P.M. Midgley (eds.)]. Cambridge University Press, Cambridge, United Kingdom and New York, NY, USA, 2013.1535 pp.

MARTÍN-VIDE, J., 2003. El tiempo y el clima. Colección Cuadernos de Medio Ambiente. Rubes Editorial. Barcelona, España. 127 pp.

MCKEE, T.B., N.J. DOESKIN \& J. KLEIST, 1993. Drought monitoring with multiple time scales. American Meteorological Society Preprints, Eighth Conference on Applied Climatology, January, Anaheim, CA, pp. 179-184.

PÉREZ, C., M. GARMENDIA \& J. GARMENDIA, 1986. Índices de persistencia de días lluviosos y de días secos en Gijón y San Sebastián. Lurralde: Investigación y espacio, 9: 77-84. 
PLISCOFF, P., F. LUEBERT, H. HILGER \& A. GUISAN, 2014. Effects of alternative sets of climatic predictors on species distribution models and associated estimates of extinction risk: A test with plants in an arid environment. Ecological Modelling, 288: 166-177.

QUINTANA, J. \& P. ACEITUNO, 2006. Trends and interdecadal variability of rainfall in Chile. In: Proceedings of 8 ICSHMO, INPE, Brazil, Foz do Iguaçu: 371-372.

QUINTANA, J. \& P. ACEITUNO, 2012. Changes in the rainfall regime along the extratropical west coast of South America (Chile): 30-43 S. Atmósfera, 25 (1):1-22.
SARRICOLEA, P., O. MESEGUERRUIZ \& J. MARTÍN-VIDE, 2014. Variabilidad y tendencias climáticas en Chile central en el período 1950-2010, mediante la determinación de los tipos sinópticos de Jenkinson y Collison. Boletín de la A.G.E. 64: 227-247.

THOMPSON, D. \& J. WALLACE, 2000. Annular modes in the extratropical circulation. Part I: Month-to-month variability. Journal of Climate, 13: 10001016. 